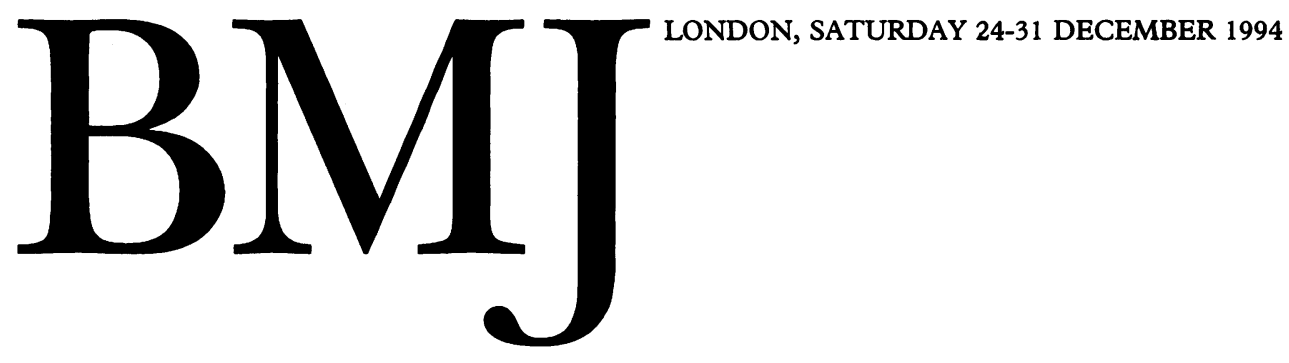

\title{
The inhumanity of medicine
}

\author{
Time to stop and think
}

\begin{abstract}
In the past few months ... I have been made aware of a large number of cases of disturbingly callous and rude behaviour by consultants and general practitioners towards patients... might I suggest that courses to remind (or perhaps teach) doctors how to behave to their patients be put high on the agenda.
\end{abstract}

This extract from a letter received recently by the editor of the $B M Y$ and the articles in a similar vein (p 1696, ${ }^{1} \mathrm{p} 1699,{ }^{2}$ p $1700^{3}$ ), raise some disturbing questions for us to ponder on over the Christmas season. The excuse that only bad news is newsworthy will not wash; such stories are becoming commonplace and encompass so much of current clinical practice that we seem to be becoming a profession of uncaring technocrats.

In seeking solutions to these problems it is important to see them in their historical perspective. Are they new? I know of no evidence that doctors of the past were so much better at handling their patients. I still vividly remember one of my first teaching rounds as a medical student, over 30 years ago. We had arrived at the end of the bed of a patient who had been found to have an inoperable lung cancer. The senior and much respected physician who was conducting the round suddenly veered away from the bed and collected together the throng of staff and students in the middle of the ward into what resembled a huddle of American football players planning their next play. The diagnosis and prognosis were discussed in hushed whispers, after which we returned to the bedside, a few banalities were exchanged, and we moved on to the next patient. Such behaviour was common during my student days. And, as so well portrayed on page 1714, for centuries journalists, cartoonists, novelists, and playwights have castigated us for our pomposity, inhumanity, and cruelty. ${ }^{4}$ Such attacks continue, as exemplified by Alan Bennett's recent play The Madness of George III.

\section{Patients are taken to the limit}

But although doctors may always have had a limited facility to treat their patients as humans, there is no doubt that the current medical scene is highlighting our deficiencies. Oncology, the basis of two articles in today's journal, is a good example. Patients are often subjected to the most intensive protocols of chemotherapy, some of which require them to be taken to death's door in an attempt to eradicate their tumours. One hundred years hence we may look back on all this in the same light as we do on bleeding and cupping today. But this is what is currently believed to be the most effective way to manage these diseases; in almost every field of modern high technology patch up practice, patients are pushed to the extremes of their endurance, and not always for reasons that include a careful appraisal of what is meant by the quality of life.

Our patients' problems are compounded by our current systems of medical care because they are not geared to support very sick people. Above all else, those with distressing chronic or terminal illnesses need continuity of care-that is, the attention and friendship of one doctor whom they can come to trust and with whom they can share their hopes and fears. Yet this kind of relationship is all too rarely available to them. Too few consultants exist to look after the increasing numbers of patients undergoing periods of intensive treatment or in their final illness.

\section{No continuity}

Because young doctors are constantly rotating through different training programmes, and as a consequence of the new regulations to limit their working hours, there is lack of day to day continuity of care at all junior grades. Patients are looked after by continuously changing teams of doctors and nurses, a pattern of care that also spills over into general practice, where the chances of them always seeing their own family doctor are equally limited.

And in the frenetic reorganised NHS doctors and nurses are spending more and more time on committees, organising their business plans and contracts, and less with their patients; managerial efficiency and an increasingly rapid turnover of patients, while they may make for impressive statistics, do not necessarily reflect a caring attitude on the part of doctors. At the same time patients and relatives are much more demanding than they used to be and, not unreasonably, expect more time and explanation of their doctors. Thus it is not surprising that the deficiencies that have dogged us for the past 2000 years are being accentuated.

To what extent are our shortcomings a reflection of the pattern of medical education? Many of the criticisms levelled at doctors are not about their clinical competence; rather, they seem to reflect a deficiency of the basic skills of handling sick people as humans, poor communication, lack of kindness, 
thoughtlessness, and, in short, all the facets of good interpersonal relationships that society has a right to demand of its doctors. Can such attitudes be taught? And even if they can, given our poor track record who is to teach them?

Concerns along these lines have led to radical revisions of medical education in several countries. ${ }^{5}$ For example, the General Medical Council suggests that there should be less emphasis on the basic sciences and more on ethics, communication skills, and the social sciences, with earlier exposure to patients and their families. ${ }^{6}$ Few people would disagree that two years spent in the company of a corpse is not the most imaginative introduction to a profession that, more than any other, needs to develop the skills of talking to distressed people.

\section{The search for rounded doctors}

On the other hand, and as argued so cogently by Downie and Charlton, ${ }^{7}$ the social sciences have their limitations with respect to understanding the needs of patients as people. An undue emphasis on one particular aspect, whether it is economics, politics, religion, or ideology, can often distort a student's view of human behaviour. And social science deals with groups, not individual people. Thus while some of these changes may help to produce more caring doctors, they may be of limited value in encouraging the broader pastoral aspects of medical care.

Undoubtedly, many of the current ills of the medical profession reflect a lack of what Downie and Charlton call "whole person understanding." This is not surprising: in Britain young people who want to become doctors have to make up their minds at the age of about 15, from which time they are, in effect, narrow specialists. They undergo five or six years of intensive and mind numbing training in medical school and then an even longer period in hospital or in general practice.

Many come from comfortable middle class homes, few will ever have been patients themselves, and even fewer will have experienced much of the world outside their immediate circle of family and friends. And although they may have developed cultural interests outside medicine, some of these will also have been goal oriented-a grade VIII in music or the like that looks well on the curriculum vitae.

From the age of 15 their time for genuine relaxation and reflection, reading, enjoying the arts, learning about the world at large, and meeting people from other walks of life will have been strictly limited. And today there is not even a compulsory period of national service to offer them the opportunity to do something different for a couple of years. It is not surprising that many of them have difficulties in adapting to the day to day needs of patients of diverse backgrounds and emotional requirements and widely differing reactions to adversity.

And, given the pressures of medicine today, there may be little time to correct these deficiencies later in their careers. Have we, albeit unwittingly, evolved an education system that, from the very beginning, is destined to leave many of its products ill equipped to deal with the multifaceted needs of sick people?

It is difficult to equate the delightful, caring, and extremely gifted young people whom one encounters on their entry to medical school with some of the horror stories described in today's journal. What on earth has exposure to a few years of the medical profession done to some of them? Clearly the issues are extremely complex. While we can certainly improve things by changing undergraduate and postgraduate training and by trying to restore our hospitals and practices from the supermarkets that they have become to places that are primarily concerned with looking after sick people, we may have to go a good deal further.

We must think again about the early years of a doctor's education. Should we be following current trends in many American schools and directing future medical students towards a much more broadly based education, encouraging them to do something completely different before entering medical school, and shortening their undergraduate courses? In other words, how can we try to ensure that our trainees are more rounded people before they enter medical school and that they remain so?

Undoubtedly a core of facts required to practise medicine, together with communication skills and an understanding of social and ethical issues, can be taught-a process that can be continued during postgraduate training. But, except by example, no medical school can teach a young person how to be understanding and caring. This can come only from experience of life. Perhaps it is this above all that we have neglected as we push our brightest young people through the hothouse of modern medical education without giving them time to reflect on what their profession is all about.

Although there are "bad eggs" in every profession, I do not believe that many doctors are inherently inhumane or uncaring. Rather, I suspect that, from the time that they decide on a career in medicine until they retire, many of them live in such an overcharged atmosphere, and one in which the demands on them are now so great, that sometimes the central reason for what they are doing - that is, the wellbeing of their patients-is forgotten. However much we do to ensure that our students of the future are better rounded and educated, our patients will not benefit unless their doctors have enough time to spend with them. This, above all, is the message that must be conveyed to health economists and governments of the future.

\section{We can do more now}

Since, clearly, problems of doctor-patient relationships have been around for hundreds of years and during times of completely different patterns of medical education, clinical practice, and social pressure, there may be only limited cause for optimism whatever we do. Sickness has such a devastating and unpredictable effect on many patients and their families that the protean goals of good pastoral care will always be difficult to attain. Could even the most caring readers of this journal put their hands on their hearts and say that they had never been tempted to avoid spending time with particularly difficult or distressing patients during the last days of their terminal illness?

As the stories in today's journal remind us, however, there is much that we can do now to put our house in order. But the indignant anger of the woman who wrote to the Times recently inquiring why, in 1994, students at a famous London teaching hospital are still instructed to ask patients "How are your waterworks?" emphasises the subtleties of the problems that lie ahead.

\section{J WEATHERALL}

University of Oxford, John Radcliffe Hospital, Oxford OX3 9DU

\footnotetext{
1 Dying for palliative care. $B M F$ 1994;309:1696-9.

2 Going private: time for change $B M 9$ 1994:309:1699-700

3 Pink G. The price of truth ethics at the bedside. $B M 7$ 1994; 309:1700-5.

4 Porter R. Dr Doubledose—a taste of one's own medicine. BMF 1994;309:1714-8.

5 Lowry S. Medical education. London: BMJ Publishing Group, 1993.

6 General Medical Council. Tomorrow's doctors. London: Kiek and Reid, 1993

7 Downie RS, Charlton B. The making of a doctor. Medical education in theory and practice. Oxford: Oxford University Press, 1992.

8 Kieman L. Plumbing the depths. Times 1994;Nov 29:19.
} 